\title{
Population structure of Lactobacillus helveticus isolates from naturally fermented dairy products based on multilocus sequence typing
}

\author{
Zhihong Sun, ${ }^{*} \dagger$ Wenjun Liu, ${ }^{\dagger} \dagger$ Yuqin Song, ${ }^{*}$ Haiyan $\mathrm{Xu},{ }^{,}$Jie Yu, ${ }^{*}$ Menghe Bilige, ${ }^{*}$ Heping Zhang, ${ }^{*} \dagger$ \\ and Yongfu Chen* ${ }^{1}$ \\ *Key Laboratory of Dairy Biotechnology and Engineering, Ministry of Education, Inner Mongolia Agricultural University, Huhhot 010018, China \\ †Synergetic Innovation Center of Food Safety and Nutrition, Jiang Nan University, Wuxi, Jiang Su 214122, China
}

\begin{abstract}
Lactobacillus helveticus is an economically important lactic acid bacterium used in industrial dairy fermentation. In the present study, the population structure of 245 isolates of $L$. helveticus from different naturally fermented dairy products in China and Mongolia were investigated using an multilocus sequence typing scheme with 11 housekeeping genes. A total of 108 sequence types were detected, which formed 8 clonal complexes and 27 singletons. Results from Structure, SplitsTree, and ClonalFrame software analyses demonstrated the presence of 3 subpopulations in the L. helveticus isolates used in our study, namely koumiss, kurut-tarag, and panmictic lineages. Most L. helveticus isolates from particular ecological origins had specific population structures.
\end{abstract}

Key words: Lactobacillus helveticus, multilocus sequence typing, population structure

\section{INTRODUCTION}

Lactobacillus helveticus is an obligate homofermentative thermophilic lactic acid bacterium (LAB), which belongs to the Lactobacillus delbrueckii phylogenetic group (Salvetti et al., 2012; Sun et al., 2014). The species is characterized as having the ability to grow at relatively high temperatures, produce high quantities of lactic acid in milk, and express a complex array of proteolytic enzymes (Slattery et al., 2010). It is widely used as a starter culture in the manufacture of Swiss-type and long-ripened Italian cheeses, such as Emmental, Grana Padano, Parmigiano Reggiano, and Provolone (Broadbent et al., 2011; Giraffa, 2014). Lactobacillus helveticus is also a stable member of the microbial community found in naturally fermented

Received November 21, 2014

Accepted January 23, 2015

${ }^{1}$ Corresponding author: nmgyfchen@126.com dairy products, such as koumiss (Sun et al., 2010b), kurut (Sun et al., 2010a), tarag (Liu et al., 2012), dairy fan (Liu et al., 2009), and qula (Bao et al., 2012). All of these naturally fermented dairy products are made primarily without the use of any commercial starter cultures; instead, natural cultures in which L. helveticus is the predominant lactic acid bacteria are used to inoculate fresh milk in the subsequent fermentations (Liu et al., 2009, 2012; Sun et al., 2010a,b; Bao et al., 2012).

Some L. helveticus isolates, including CM4, DPC4571, CPN4, CP790, CNRZ32, and H9, are regarded as probiotics and used in the production of fermented drinks, which have properties associated with a reduction in blood pressure following ingestion (Nakamura et al., 1995; Yamamoto et al., 1999; Kilpi et al., 2007; Chen et al., 2014). This is due to the production of significant levels of the bioactive tripeptides, Val-Pro-Pro and Ile-Pro-Pro, which inhibit angiotensin I-converting enzyme (Wakai et al., 2013). Lactobacillus helveticus is an amino acid auxotrophic bacterium and has a strong proteolytic system. During milk fermentation by $L$. helveticus, it is likely that alongside the production of essential amino acid by proteolytic system, Val-Pro-Pro and Ile-Pro-Pro are also formed (Wakai et al., 2013).

Despite the common use of L. helveticus in the dairy industry, its genetic diversity and population structure are poorly understood. Elucidating the genetic diversity and phylogenetic relationships among isolates would help in understanding the evolution of ecological and biological properties of those isolates, and contribute to optimizing their industrial exploitation (Diancourt et al., 2007). Previous studies have shown that several DNA fingerprinting techniques were able to distinguish different genotypes within of L. helveticus. These include randomly amplified polymorphic DNA (Aquilanti et al., 2010), repetitive sequence PCR (Jensen et al., 2009), ribotyping (Giraffa et al., 2000), and pulsed-field gel electrophoresis (Jenkins et al., 2002). However, these techniques are not sufficient for isolate delineation and phylogenetic inference (Achtman, 2002).

Multilocus sequence typing (MLST), a method for studying microbial population biology based on analyz- 
ing the nucleotide and allelic polymorphism of a set of 6 to 10 housekeeping genes, was developed by Maiden et al. (1998). Recently, MLST has been used to elucidate the evolution and population structure of several Lactobacillus species, including the L. acidophilus complex (Ramachandran et al., 2013), L. casei (Cai et al., 2007; Diancourt et al., 2007), L. delbrueckii (Tanigawa and Watanabe, 2011), L. plantarum (de Las Rivas et al., 2006), L. reuteri (Su et al., 2012), L. sakei (Chaillou et al., 2013), and L. sanfranciscensis (Picozzi et al., 2010), but it has not yet been applied to L. helveticus.

To better understand the evolutionary adaptation of $L$. helveticus to different ecological niches, we developed an MLST scheme and investigated evolutionary characteristics and population structure of 245 isolates from different naturally fermented dairy products in China and some areas of Mongolia.

\section{MATERIALS AND METHODS}

\section{Bacterial Isolates and DNA Extraction}

Two hundred forty-five isolates of L. helveticus were used in this study (Supplemental Table S1, http:// dx.doi.org/10.3168/jds.2014-9133). Of these, 238 were obtained from the Lactic Acid Bacteria Collection Centre of the Inner Mongolia Agriculture University of China. They were isolated from various naturally fermented dairy products, including koumiss (64), tarag (84), kurut (45), qula (20) hormog (8), and dairy fan (17), in 7 provinces of China and 13 provinces of Mongolia (Supplemental Table S1). Sequences of the type strain of $L$. helveticus DSM $20075^{\mathrm{T}}$ and 6 reference strains were obtained directly from the National Center for Biotechnology Information genome database (http://www.ncbi.nlm.nih.gov/genome/672). Lactobacillus helveticus strains were grown overnight at $37^{\circ} \mathrm{C}$ in $5 \mathrm{~mL}$ of MRS broth (Oxoid, Cambridge, UK). Total genomic DNA was extracted from cultures as described by Sun et al. (2010a).

\section{Selection of MLST Loci}

General criteria for selecting genes for the MLST analysis include the even separation across the chromosome, functions of the encoded proteins, presence in all the strains as a single copy, and a size of at least $1 \mathrm{~Kb}$ (Cai et al., 2007). Based on these criteria and by comparing the gene sequences of L. helveticus DPC 4571 (CP000517), H9 (CP002427), and H10 (CP002429), 11 housekeeping genes, $\operatorname{clp} X$, dnaA, dnaK, groEL, murC, murE, pepN, pepX, pyrG, recA, and uvrC gene, were selected for MLST analysis. The PCR primers for the 11 housekeeping genes were designed using Primer Premier 6.0 software (Premier Biosoft International, Palo Alto, CA) on the basis of the genome sequence of $L$. helveticus H10 (Zhao et al., 2011; Table 1).

Table 1. The information on the genes and primers used for MLST

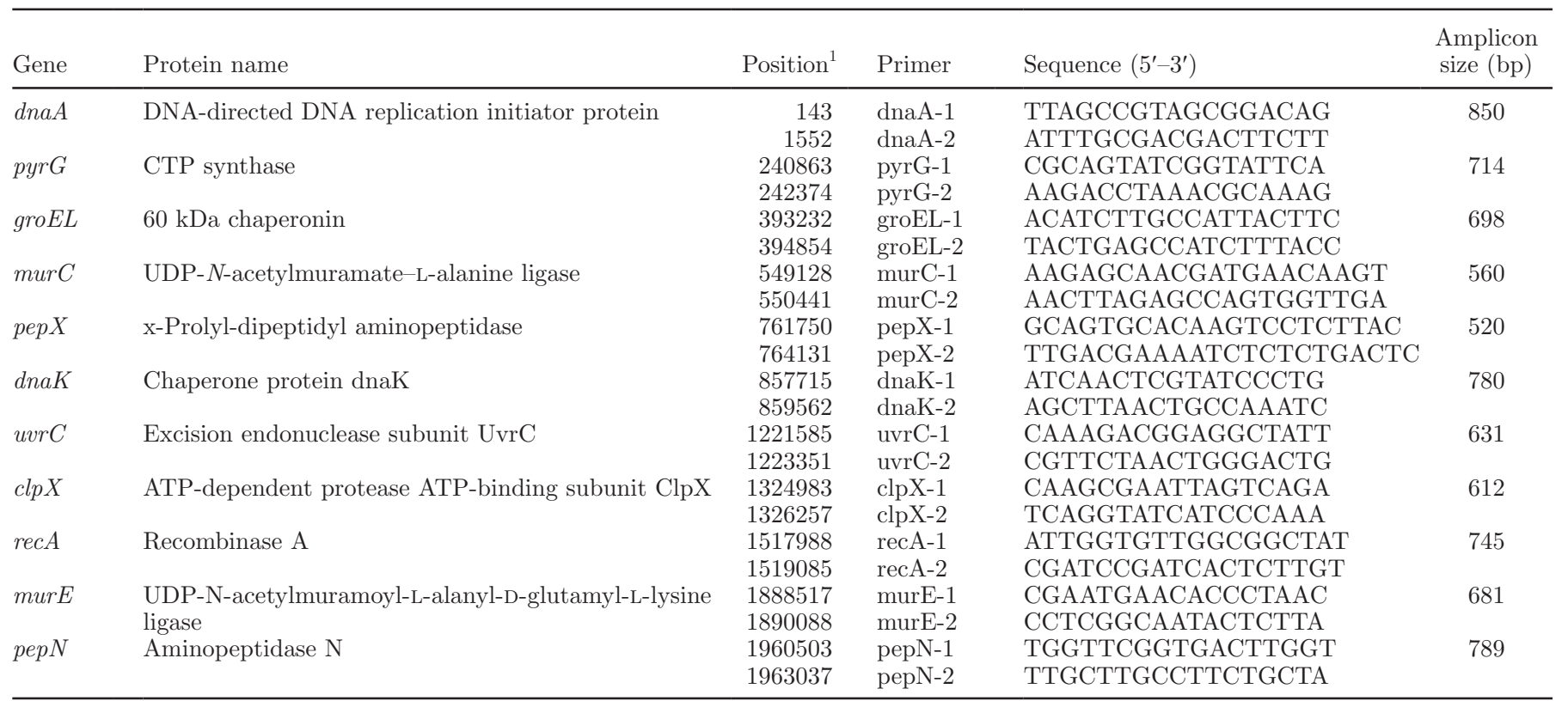

${ }^{1}$ Numbers denote the positions of the first and last bases of the gene on the L. helveticus H10 chromosome. 


\section{PCR Amplification and DNA Sequencing}

The PCR amplifications of MLST loci were done with proofreading Pyrobest DNA Polymerase (TaKaRa, Dalian, China) and carried out on an automatic thermal cycler (PTC-200, MJ Research, Waltham, MA). The PCR mixture $(50 \mu \mathrm{L})$ for each target contained 50 ng of genomic DNA, $20 \mathrm{~m} M$ of each dNTP, 10 pmol of primer set, $1 \mathrm{U}$ Pyrobest DNA polymerase in $1 \times$ Pyrobest buffer II (with $\mathrm{Mg}^{2+}$ ). The PCR conditions began with an initial denaturation step of 4 min at $95^{\circ} \mathrm{C}$, followed by 30 cycles of $30 \mathrm{~s}$ denaturation at $95^{\circ} \mathrm{C}, 1 \mathrm{~min}$ annealing at temperature of each locus for 1 min elongation at $72^{\circ} \mathrm{C}$, and ended with a final elongation step of $72^{\circ} \mathrm{C}$ for $8 \mathrm{~min}$. The PCR products were individually checked after agarose gel electrophoresis and subsequently cleaned from residual primers using a QIAquick 96 PCR purification kit (Qiagen, Hilden, Germany). The purified PCR products were sequenced by the Tianjin Biochip Corporation. The same primers from the PCR amplifications were used for sequencing on both DNA strands.

\section{Descriptive Analysis of MLST Sequence Data}

Sequences were trimmed to a uniform length for each gene, which was determined from multiple alignment of all sequences. The forward and reverse sequences were trimmed, aligned, and analyzed using Bionumerics 6.6 software (Applied Maths, Sint-Martens-Latem, Belgium) and each unique sequence was assigned a different allele number. Different allelic sequences (with at least one nucleotide difference) were assigned arbitrary numbers (Laukkanen-Ninios et al., 2011). For each $L$. helveticus strain, the combination of 11 alleles defined its allelic profile, and a unique allelic profile was designated a sequence type (ST).

The mean GC content of the DNA and the $d_{\mathrm{N}} / d_{\mathrm{S}}$ ratios (where $d_{\mathrm{N}}$ is the number of nonsynonymous substitutions per nonsynonymous site and $d_{\mathrm{S}}$ is the number of synonymous substitutions per synonymous site) were determined using Start v2.0 (Jolley et al., 2001). The number of polymorphic sites, the nucleotide diversity $(\pi)$ per site, and Tajima's $D$ test were calculated using DnaSP 5.0 (Rozas et al., 2003). The standardized index of association $\left(\boldsymbol{I}_{A}^{S}\right)$ was calculated to determine the degree of linkage disequilibrium between alleles using LIAN 3.6 (Haubold and Hudson, 2000). The phi test for recombination based on individual loci of the whole strain collection was done with SplitsTree4 (Huson and Bryant, 2006). This test was able to produce a $P$-value of the pairwise homoplasy index test (phi, a cutoff of
0.05 was used herein) to determine the DNA regions exhibiting strongest evidence of mosaicism.

\section{Population Analysis}

The software Structure 2.3 (Falush et al., 2007) with an admixture model was used to infer the lineage ancestry of the unique ST assuming that each ST had derived all of its ancestry from $\mathrm{K}$ ancestral subpopulations. Three individual runs per value of $\mathrm{K}$ (chosen between 3 and 10) were performed using 200,000 burn-in iterations and 300,000 sampling iterations. The $\mathrm{K}$ value that generated the highest median posterior probability was used as the probable number of ancestral populations.

The relationships between individual ST and clonal complexes (CC) were analyzed using eBURST 3.0 (Based Upon Related Sequence Types) software (http:// eburst.mlst.net/; Feil et al., 2004). The relationships between the ST and the region and original sources of all isolates were determined using a minimum spanning tree (MSTree) analysis performed with Prim's algorithm in the BioNumerics 6.6 (Applied-Maths, Sint Maartens-Latem, Belgium).

\section{Phylogenetic Analysis and Recombination}

The phylogenetic trees from the concatenated sequences $(5,102 \mathrm{bp})$ were constructed by the neighborjoining (NJ) and maximum-likelihood (ML) methods using Mega 5.0 (http://www.megasoftware.net), with 1,000 replicates in bootstrap analysis. To evaluate the degree of tree-like structure for alleles of each locus and all ST, a split decomposition method was applied using SplitsTree 4.0 (Huson and Bryant, 2006).

The phylogenetic and evolutionary relationships among of $L$. helveticus strains were inferred using ClonalFrame 1.2 (Didelot and Falush, 2007). Three independent runs were performed for each analysis, with a burn-in cycle of the Markov chain Monte Carlo algorithm fixed to 100,000 iterations and a posterior sampling of 100,000 iterations. The prior iterations were discarded and model parameters were sampled in the second period of the run at every 100 iterations. From this run, several measures were also taken such as $\rho / \theta$ (relative frequency of occurrence of recombination and mutation) and $r / m$ (relative effect of recombination and mutation in the diversification). The genealogy of the population was summarized and the robustness of the tree topology was evaluated by concatenating the posterior samples of the 3 runs to build up a $50 \%$ majority rule consensus tree using the ClonalFrame GUI. 
Table 2. Nucleotide and allelic diversity of the multilocus sequence typing loci

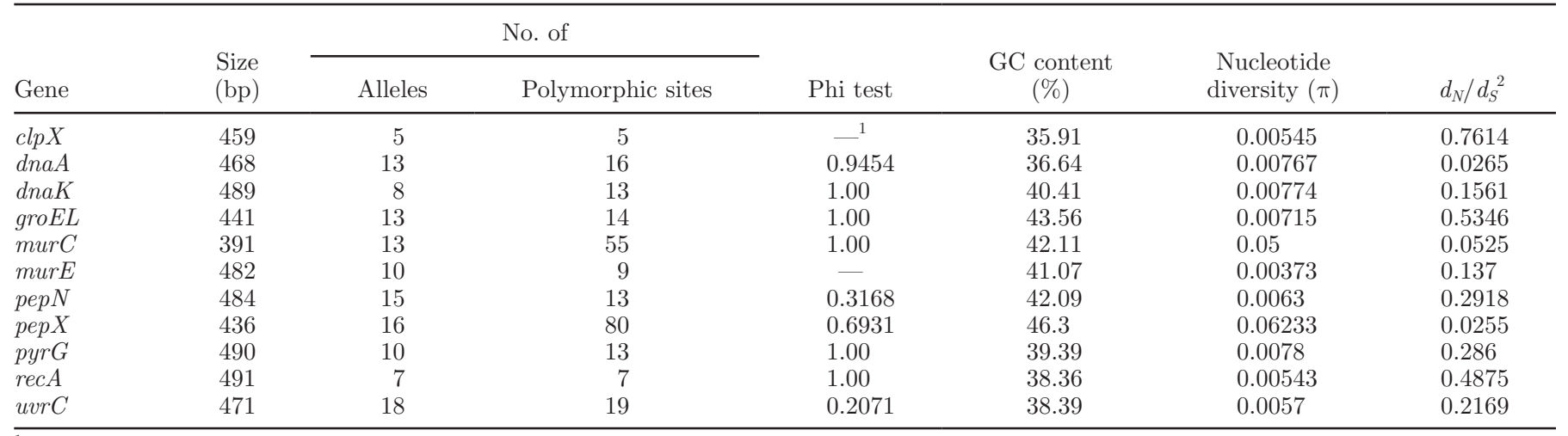

1- indicates too few informative characters to use the phi test as implemented here.

${ }^{2}$ Where $d_{\mathrm{N}}$ is the number of nonsynonymous substitutions per nonsynonymous site, and $d_{\mathrm{S}}$ is the number of synonymous substitutions per synonymous site.

\section{Nucleotide Sequence Accession Numbers}

Sequences of the 11 MLST loci were deposited in the GenBank library under accession numbers KP028043 to KP030660.

\section{RESULTS}

\section{Diversity Analysis of Nucleotide Sequences}

We developed a new MLST scheme for L. helveticus based on fragments of 11 housekeeping genes. Three genes, $\operatorname{pyr} G$, groEL, and $\operatorname{rec} A$, were used in previous analysis of $L$. delbrueckii (Tanigawa and Watanabe, 2011). Others include 5 genes (dnaA, murC, pepX, $d n a K$, and $\operatorname{clp} X)$ that were widely used in bacterial analysis (http://pubmlst.org/) plus 3 genes, murE, pepN, and uvrC.

The trimmed sequences of these housekeeping gene fragments ranged in length from 391 to $491 \mathrm{bp}$, with a total of $5,102 \mathrm{bp}$ in the concatenated sequences (Table 2). The mean GC content varied from $35.91 \%$ $(c l p X)$ to $46.30 \%(p e p N)$, compared with $36.92 \%$ in the whole L. helveticus genome (Table 2). Among the 11 loci, the number of alleles ranged from $5(\operatorname{clp} X)$ to 18 (uvrC), and the number of polymorphic sites ranged from $5(\operatorname{clp} X)$ to $80($ pep $X$; Table 2$)$. A total of 244 polymorphic sites were detected in the 245 isolates of L. helveticus evaluated, which formed 108 sequence types. The nucleotide diversity site $(\pi)$, which is defined as the average number of nucleotide differences per site between 2 randomly selected sequences, varied from 0.00373 (murE) to 0.06233 (pepX; Table 2). The ratio of mean nonsynonymous substitutions to mean synonymous substitutions $\left(d_{\mathrm{N}} / d_{\mathrm{S}}\right)$ for the 11 loci varied between $0.0255(p e p X)$ and 0.7614 ( $c l p X$; Table 2),
Values of $d_{\mathrm{N}} / d_{\mathrm{S}}$ were lower than 1 , indicating negative selection on the selected genes.

\section{Defining ST and Their Distribution Within Multiple Clonal Complexes}

The ST number was assigned based on the allele profiles for the 11 MLST genes (Supplemental Table S2; http://dx.doi.org/10.3168/jds.2014-9133). We identified 108 different ST among the 245 L. helveticus strains (Supplemental Table S2). Sixty-six ST were represented by only one strain, whereas $26 \mathrm{ST}$ contained at least 3 strains. Of these, ST- 1 was the largest and contained 21 isolates, followed by ST-32 (12 strains), ST-7 (10 strains), ST-70 (9 strains), and ST-27 (8 strains); the remaining ST had fewer than 7 isolates.

A CC is defined as a group of ST in which 2 connected ST differ by 1 locus, and can be used to measure the relatedness of isolates (Feil et al., 2004). A snapshot of the population structure was obtained using eBURST analysis on the basis of allele profiles. Among the 108 ST identified from the 245 L. helveticus strains, 27 were singletons and the rest fell in to $8 \mathrm{CC}$ (Figure 1; Supplemental Figure S1 and Supplemental Table S1, http://dx.doi.org/10.3168/jds.2014-9133). The CC27 (named after its founder ST) was the largest and contained 32 ST representing 74 L. helveticus strains, $54 \%$ of which were isolated from Tarag in Mongolia. The CC7 contained $25 \mathrm{ST}$ representing 84 L. helveticus strains, $78 \%$ of which were isolated from dairy fan (10 strains), koumiss (14 strains), kurut (7 strains), qula (10 strains), and tarag (19 strains) in China. The CC21 contained 5 isolates from koumiss and 4 isolates from tarag in Inner Mongolia, China. The CC63, CC67, and CC46 contained isolates exclusively from koumiss from Xinjiang, kurut from Qinghai, China, and tarag from 


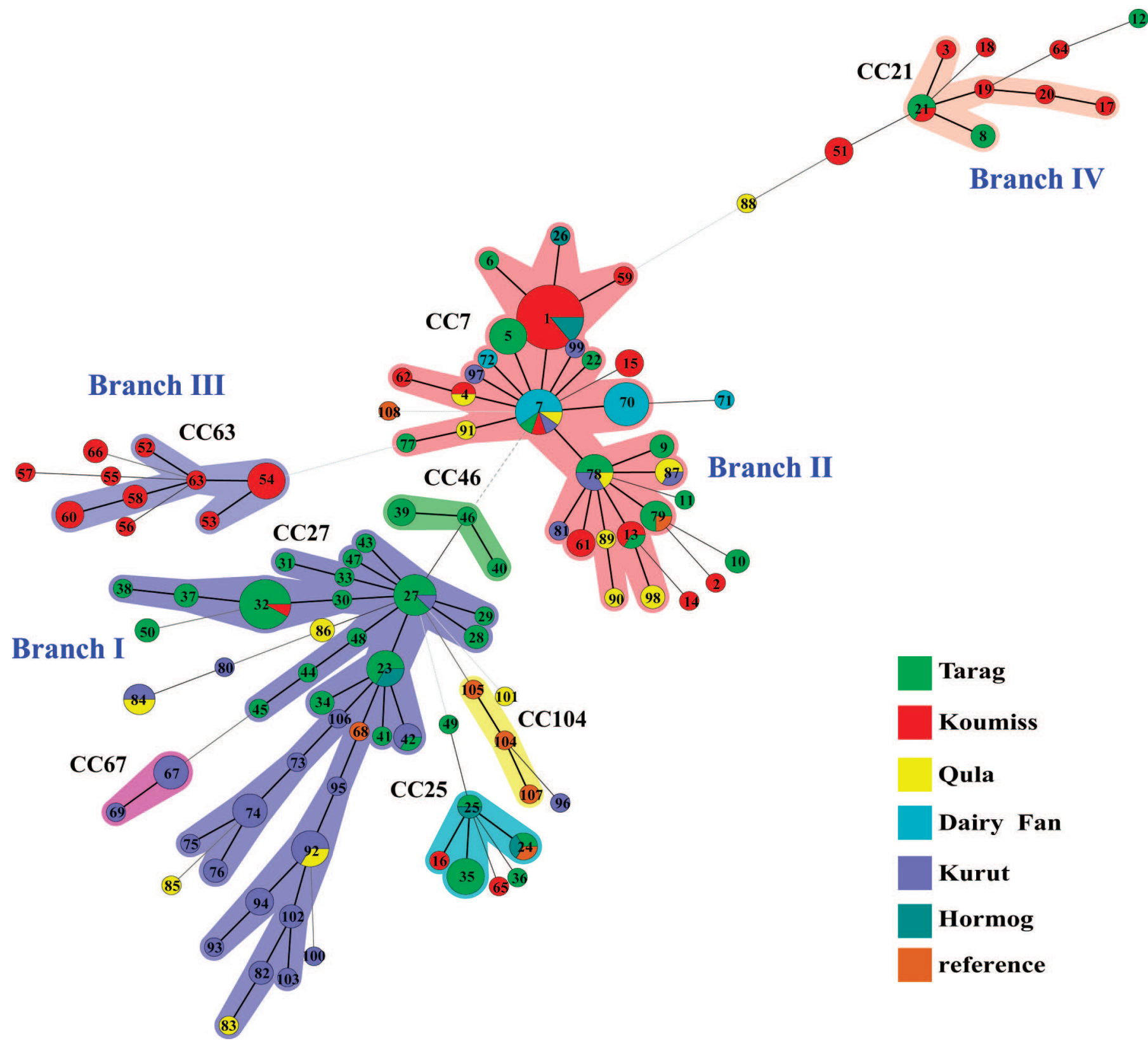

Figure 1. Minimum spanning tree analysis of the 245 Lactobacillus helveticus strains from different dairy products based on the allelic profiles of 11 genes. Each circle corresponds to a sequence type (ST), and the circle size corresponds to the number of strains sharing the same ST. The shaded zones between particular groups of circles indicate that these profiles belong to the same clonal complex (CC). The strength of the link (bold, plain, or discontinuous) is correlated to the genetic similarity between profiles.

Mongolia, respectively. The CC104 contained only the 3 reference strains, L. helveticus DSM20075 ${ }^{\mathrm{T}}$, CNRZ32, and MTCC5463.

In the MSTree, the $245 \mathrm{~L}$. helveticus strains studied were clustered into 4 main branches (Figure 1 and Supplemental Figure S2; http://dx.doi.org/10.3168/ jds.2014-9133). Relationships between the clusters, their source dairy product, and geographical region are presented. Branch I was composed of $5 \mathrm{CC}$ and 11 singletons, representing $112 \mathrm{~L}$. helveticus isolates and 4 reference strains; most of the isolates $(82 \%)$ were from kurut (38) and tarag (57). Branch II consisted of CC7 and 7 singletons. Branch III and Branch IV was mainly composed of isolates from koumiss, particularly in Xinjiang and Inner Mongolia (e.g., CC63 and CC21; Supplemental Figure S2). Overall, the MSTree revealed 
that $L$. helveticus isolates were clustered into several branches associated with the dairy products from which they were isolated.

\section{Population Structure and Phylogenetic Relationships}

To investigate the relatedness among the $L$. helveticus strains and to gain deeper insights into the genetic structure of the species, NJ- and ML-based phylogenetic trees were built from the concatenated sequences of the 108 ST. The topology of the NJ tree (Figure 2A) was congruent with that of the ML tree (Supplemental Figure S3; http://dx.doi.org/10.3168/jds.2014-9133), except for 7 ST. As shown in Figure 2A, 3 distinct lineages, termed lineage 1 to lineage 3 , were observed with $100 \%$ of bootstrap support. This result was consistent with the 4 branches of the MSTree, with the exception of ST59 and ST77. Furthermore, branch III and branch IV were integrated into lineage 3, which comprised mostly L. helveticus isolates from koumiss. Although most branches within all the 3 lineages were poorly supported by bootstrap values, the bootstrap values of the main clades exceeded 80 , which means that the main topology was robust.

The linkage model of Structure was applied to the sequence data set of $108 \mathrm{ST}$. Multiple runs with K values from 2 to 10 showed maximal posterior probability at $\mathrm{K}=3$. The $108 \mathrm{~L}$. helveticus $\mathrm{ST}$ were divided into 3 distinct subpopulations based on the major ancestral source of genetic diversity of each ST (Figure 2B), corresponded to the 3 lineages from the NJ tree. Little admixture was found of ancestral sources between these 3 subpopulations, suggesting high homogeneity of the ST of each subpopulation. In addition, the split network of the 108 ST also revealed 3 distinct subpopulations corresponding to lineage 1 to lineage 3 (Figure 3 ).

A 50\% consensus tree constructed using ClonalFrame identified similar subgroups as the NJ tree and split decomposition analysis (Figure 4). The 3 subpopulations identified by Structure (Figure 2B) corresponded to the clades identified in Figure 4 (represented by corresponding colors). These observations provide strong evidence for the presence of 3 lineages among these $L$. helveticus isolates, associating with particular ecological niches, namely a koumiss niche, a kurut-tarag niche, and a panmictic niche.

\section{Detecting Evidence for Recombination}

The multilocus linkage disequilibrium between the MLST loci was measured using $I_{A}{ }^{S}$. An $I_{A}{ }^{S}$ of 0.1948 $(P=0.000)$ was obtained for the 11 loci. This value was significantly different from zero, indicating a ten- dency of linkage disequilibrium between the alleles at the level of the whole population. From the split graphs derived from split decomposition analysis, we can see that few parallelogram structures were formed for $\operatorname{clp} X$, $d n a A$, groEL, pepN, pepX, and uvrC, indicating that these genes had undergone little intergenic recombination during the evolution (Supplemental Figure S4; http://dx.doi.org/10.3168/jds.2014-9133). In contrast, the concatenated sequences of the 11 loci displayed a complex network-like structure (Figure 3), suggesting that several recombination events had occurred in the 11 loci based on the phi test $(P=4.266 \mathrm{E}-11)$.

To further elucidate the likelihood of recombination, analysis was performed using ClonalFrame on the concatenated sequences of all $L$. helveticus strains. We found only limited proportion of recombination events in the $245 \mathrm{~L}$. helveticus strains evaluated, and a low value for $r / m(r / m=0.35,95 \%$ credibility interval $0.08-0.75)$ and $\rho / \theta(\rho / \theta=0.02,95 \%$ credibility interval $0.002-0.055)$, and indicating that genetic variation at these loci may be attributed less to recombination.

\section{Missense Mutations in pepX}

The pep $X$ gene encodes the $\mathrm{x}$-prolyl-dipeptidyl aminopeptidase, which specifically hydrolyzes a peptide bond after X-Pro dipeptide and releases the amino-terminal X-Pro dipeptide (Kimura et al., 2002). Nine missense mutation sites were found in the pep $X$ gene of all strains at nucleotide positions $5,15,21,303,369,372,426$, 429 , and 432 , respectively, which attributed to 5 alleles. Interestingly, 7 of them could be found in all $21 \mathrm{ST}$ (35 strains) only from lineage 3 . In other words, most of the $L$. helveticus isolates from koumiss inherited 7 kinds of missense mutation. It was speculated that the pepX gene missense mutations might have a positive effect on relevant phenotypes, thus increasing the fitness of the lineage 3 organisms in koumiss.

\section{DISCUSSION}

As functional fermented dairy products continue to increase in popularity, more and more Lactobacillus strains are being used as probiotics. We revealed the population structure and phylogenetic relationships among $245 \mathrm{~L}$. helveticus isolates from different naturally fermented dairy products in different regions (7 provinces of China and 13 provinces of Mongolia) using a MLST scheme based on 11 housekeeping gene fragments.

The low $d_{\mathrm{N}} / d_{\mathrm{S}}$ ratio obtained for all 11 genes suggested that purifying selection had been dominant in these housekeeping genes. This finding was consistent with the conclusions of other studies of lactic acid bac- 


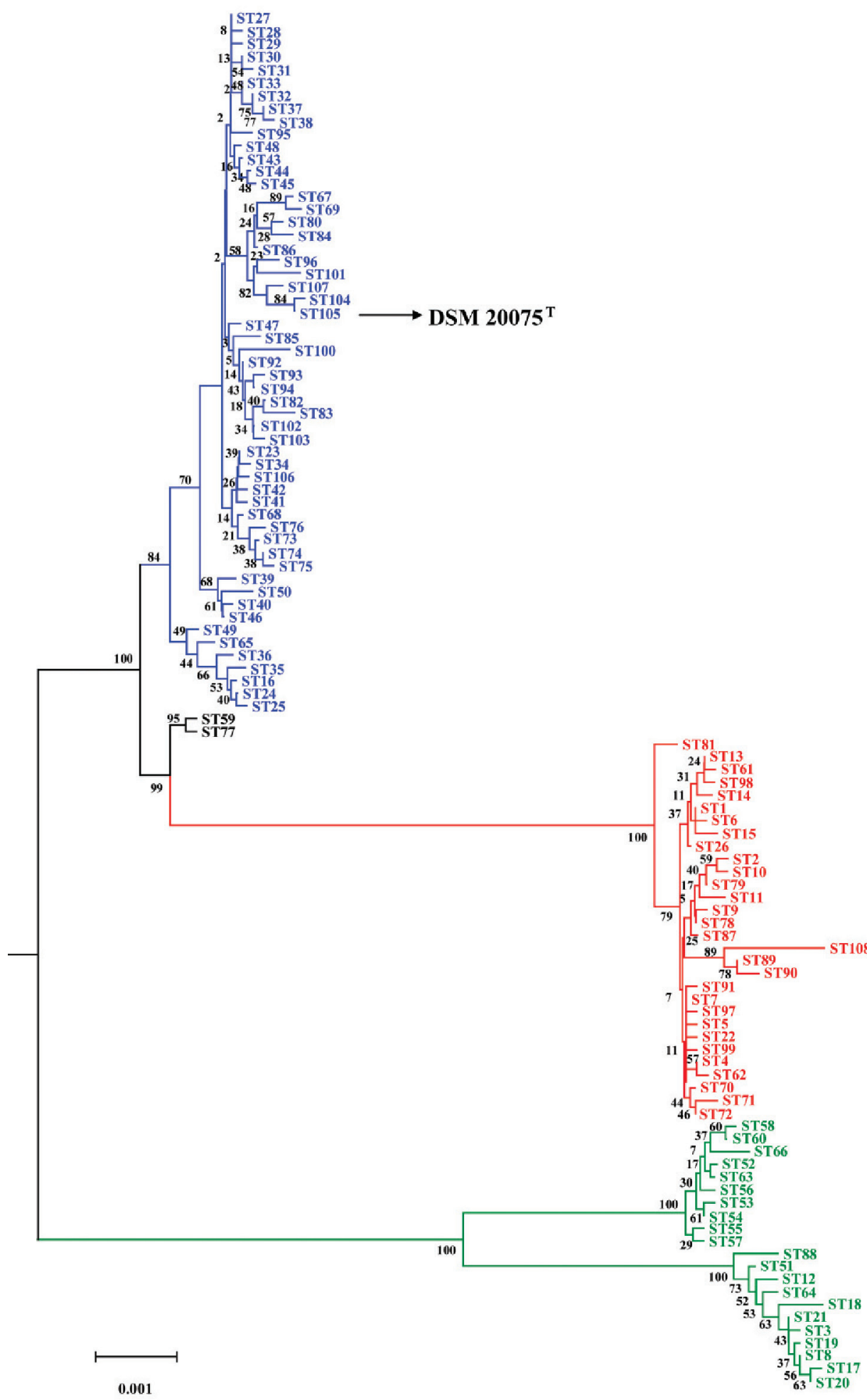

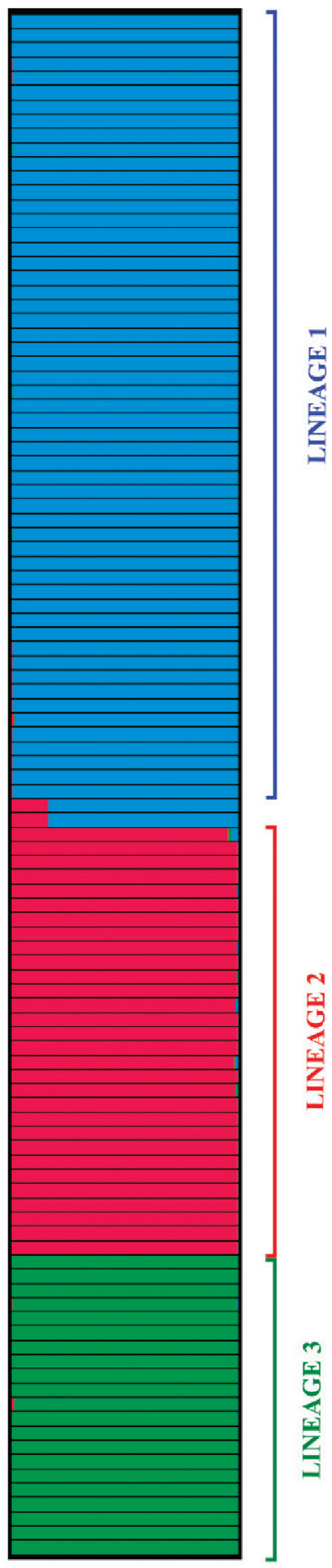

(B) Lineage of population

(A) Phylogenetic NJ tree

Figure 2. Ancestry and neighbor-joining (NJ) tree of 108 Lactobacillus helveticus sequence types (ST). (A) Phylogenetic NJ tree obtained from the concatenated sequences from the 108 ST. Bootstrap values are indicated for all branches. The ST are colored according to their affiliation to 1 of the 3 lineages; admixed ST are in black. (B) Proportion of genetic material derived from each of 3 subpopulations for each ST as inferred by STRUCTURE (assuming $\mathrm{K}=3$ populations and applying the linkage model). Ancestral subpopulations are colored in blue (lineage 1), red (lineage 2), and green (lineage 3), respectively. Each bar (stacked vertically) represents 1 of 108 ST, ordered on the y-axis by their positions in the NJ tree. 


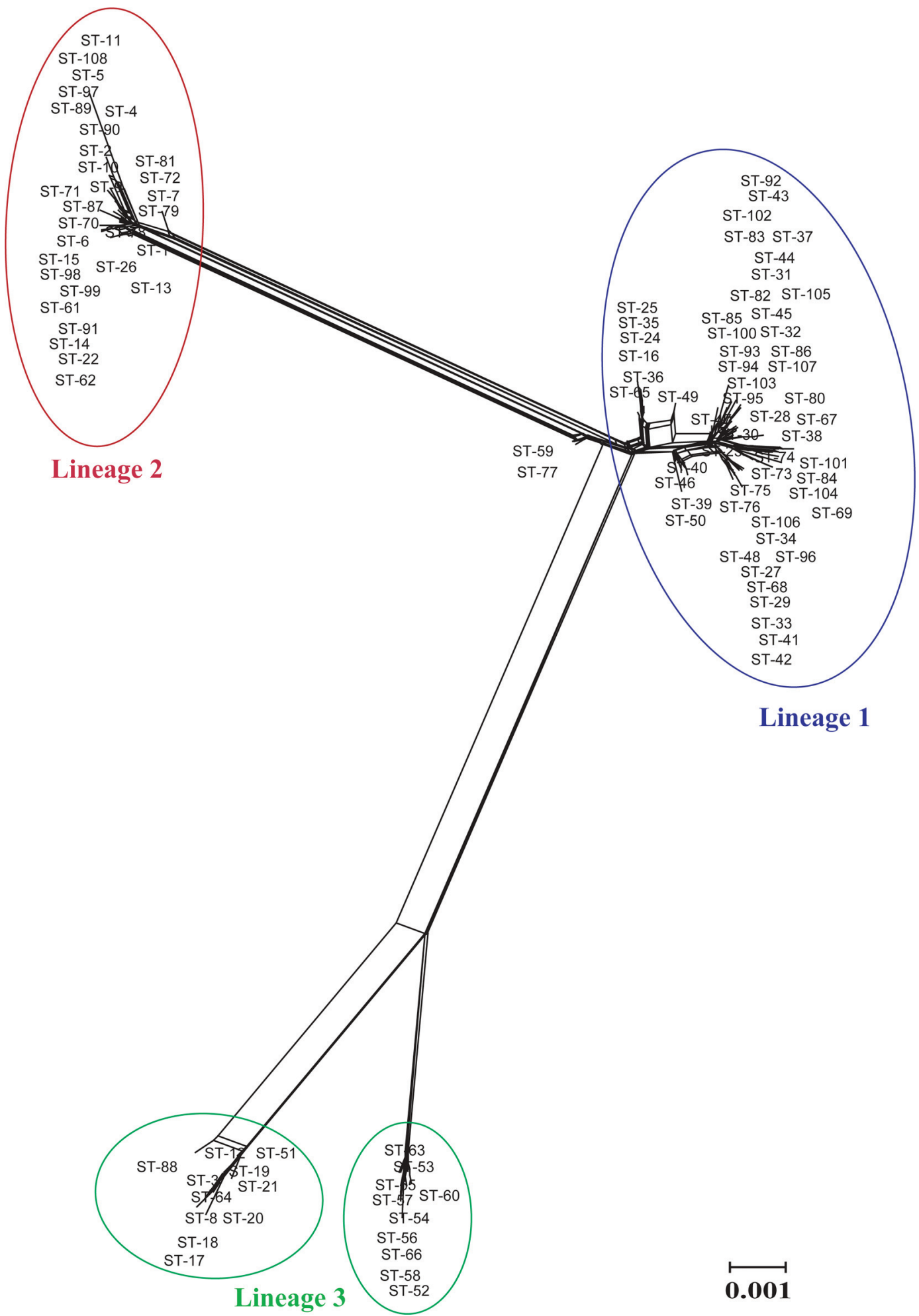

Figure 3. Split decomposition analysis based on all 11 multilocus sequence typing loci. Color version available online.

teria (de Las Rivas et al., 2006; Diancourt et al., 2007; Picozzi et al., 2010; Xu et al., 2014). A total of 108 ST with an average of 12 alleles per locus were identified in the current study, indicating a high degree of genotypic diversity at slowly evolving loci. Although pep $X$ had the highest degree of nucleotide diversity (0.06233) and 


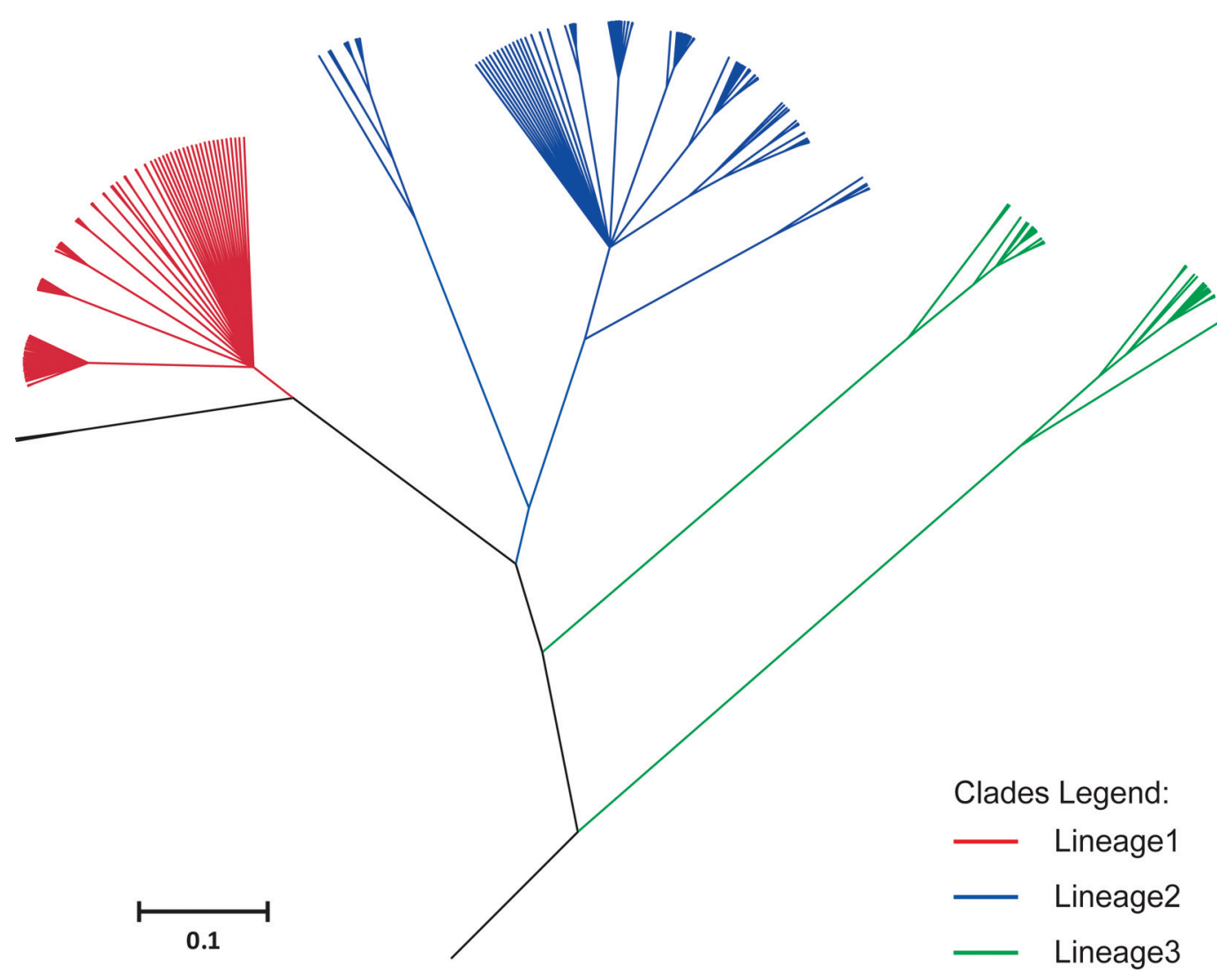

Figure 4. Clonal genealogy inferred by ClonalFrame from the 245 Lactobacillus helveticus strains. The populations identified in Figure 2 correspond to the clades identified here in the ClonalFrame clonal genealogy and have, therefore, been colored with the same colors as in Figure 2.

the greatest number of polymorphic nucleotide sites (80), it only had 16 alleles in all the 245 L. helveticus strains evaluated. Previous studies on the pep $X$ gene of Lactococcus lactic have also similar results; they found high levels of nucleotide diversity (0.07644) but had only 15 alleles (Xu et al., 2014).

Previous studies using MLST grouped a collection of $40 \mathrm{~L}$. casei isolates into clusters associated with the dairy product from which they had been isolated; they identified a cheese cluster, a silage cluster, and a mixed cluster, the latter primarily containing isolates from the human gastrointestinal tract but also some isolates from cheese (Cai et al., 2007). Correlations were also observed within the MSTree they produced confirming that their study demonstrated specificity to particular ecological niches (Cai et al., 2007). In our study, we found similar results and could associate particular branches of isolates with the dairy product from which they were isolated. Branch III and branch IV contained isolates from koumiss, which we suggest could be named the koumiss group, and branch I contained isolates from kurut and tarag, which we suggest could be named the kurut-tarag group. Branch II was a panmictic group, including isolates from a range of origins, including koumiss, kurut, tarag, hormog, qula, and dairy fan. This last group may occur due to the versatility of individual $L$. helveticus isolates, which can survive, or even grow, in a range of different ecological niches (de Las Rivas et al., 2006). Therefore, our results indicate that different ecological niches may play an important role in the evolution of $L$. helveticus strains because genetic relationships are generally correlated with ecological niches. Similarly, 2 subpopulations had been observed in the strains of Oenococcus oeni, which reflected adaptations of 2 different ecological niches (Bridier et al., 2010).

It is widely accepted that recombination plays a driving role in the evolution of most bacteria (Didelot and Maiden, 2010). According to the well-documented knowledge on the genome structure and arrangement of L. helveticus, recombination events are likely to be the main evolutionary drivers as indicated by the presence of multiple IS sequences (Callanan et al., 2008; Cremonesi et al., 2012). The plasticity of the L. helveticus genome is well documented also by the wide presence of CRISPR loci within its genome (Kaleta et al., 2010). In contrast, our results using ClonalFrame to study recombination in $245 \mathrm{~L}$. helveticus isolates showed low 
recombination rates $(r / m=0.35)$ and a low relative frequency of recombination compared with point mutation $(\rho / \theta=0.02)$, indicating that recombination may have happened. However, it has not played a major role in evolution of these loci. Whether this discrepancy between our data and the literature could be explained by the rather different ecological niches and geographical areas where the strains were isolated from still needs to be explored.

\section{CONCLUSIONS}

This is the first report of genetic diversity and population structure of $L$. helveticus isolated from different naturally fermented dairy products based on a developed MLST scheme. In this study, we analyzed the genetic diversity, population structure, recombination, and adaptation of the pepX gene in 245 L. helveticus strains, and identified 3 separate lineages; namely the koumiss, kurut-tarag, and panmictic lineages, that were related to specific ecological niches. Further, future whole-genome sequencing study including more isolates from other sources will give a much deeper understanding of the genetic diversity, phylogeny, and population structure of $L$. helveticus.

\section{ACKNOWLEDGMENTS}

This research was supported by the National Natural Science Foundation of China (Beijing, China; 31101315), International S\&T Cooperation Program of China (Beijing, China; ISTCP, 2014DFR31150), Hi-Tech Research and Development Program of China (Beijing, China; 863 Program, 2011AA100901 and 2011AA100902), and the Special Fund for Agro-Scientific Research in the Public Interest (Beijing, China; 201303085). We thank Kwok Lai Yu (Key Laboratory of Dairy Biotechnology and Engineering, Ministry of Education, Inner Mongolia Agricultural University, Huhhot, Inner Mongolia, China) for her critical reading of the manuscript.

\section{REFERENCES}

Achtman, M. 2002. A phylogenetic perspective on molecular epidemiology. Pages 485-509 in Molecular Medical Microbiology. Vol. 1. M. Sussman, ed. Academic Press, London, UK.

Aquilanti, L., A. Carbini, R. Strappati, S. Santarelli, G. Silvestri, C. Garofalo, and F. Clementi. 2010. Characterisation of Lactobacillus helveticus strains producing antihypertensive peptides by RAPD and inverse-PCR of IS elements. Benef. Microbes 1:229-242.

Bao, Q., W. Liu, J. Yu, W. Wang, M. Qing, X. Chen, F. Wang, J. Zhang, W. Zhang, J. Qiao, T. Sun, and H. Zhang. 2012. Isolation and identification of cultivable lactic acid bacteria in traditional yak milk products of Gansu Province in China. J. Gen. Appl. Microbiol. 58:95-105.

Bridier, J., O. Claisse, M. Coton, E. Coton, and A. Lonvaud-Funel. 2010. Evidence of distinct populations and specific subpopulations within the species Oenococcus oeni. Appl. Environ. Microbiol. 76:7754-7764.

Broadbent, J. R., H. Cai, R. L. Larsen, J. E. Hughes, D. L. Welker, V. G. De Carvalho, T. A. Tompkins, Y. Ardo, F. Vogensen, A. De Lorentiis, M. Gatti, E. Neviani, and J. L. Steele. 2011. Genetic diversity in proteolytic enzymes and amino acid metabolism among Lactobacillus helveticus strains. J. Dairy Sci. 94:4313-4328.

Cai, H., B. T. Rodriguez, W. Zhang, J. R. Broadbent, and J. L. Steele. 2007. Genotypic and phenotypic characterization of Lactobacillus casei strains isolated from different ecological niches suggests frequent recombination and niche specificity. Microbiology 153:2655-2665.

Callanan, M., P. Kaleta, J. O'Callaghan, O. O'Sullivan, K. Jordan, O. McAuliffe, A. Sangrador-Vegas, L. Slattery, G. F. Fitzgerald, T. Beresford, and R. P. Ross. 2008. Genome sequence of Lactobacillus helveticus, an organism distinguished by selective gene loss and insertion sequence element expansion. J. Bacteriol. 190:727-735.

Chaillou, S., I. Lucquin, A. Najjari, M. Zagorec, and M. C. Champomier-Verges. 2013. Population genetics of Lactobacillus sakei reveals three lineages with distinct evolutionary histories. PLoS ONE 8:e73253.

Chen, Y., W. Liu, J. Xue, J. Yang, X. Chen, Y. Shao, L. Y. Kwok, M. Bilige, L. Mang, and H. Zhang. 2014. Angiotensin-converting enzyme inhibitory activity of Lactobacillus helveticus strains from traditional fermented dairy foods and antihypertensive effect of fermented milk of strain H9. J. Dairy Sci. 97:6680-6692.

Cremonesi, P., S. Chessa, and B. Castiglioni. 2012. Genome sequence and analysis of Lactobacillus helveticus. Front. Microbiol. 3:435.

de Las Rivas, B., A. Marcobal, and R. Munoz. 2006. Development of a multilocus sequence typing method for analysis of Lactobacillus plantarum strains. Microbiology 152:85-93.

Diancourt, L., V. Passet, C. Chervaux, P. Garault, T. Smokvina, and S. Brisse. 2007. Multilocus sequence typing of Lactobacillus casei reveals a clonal population structure with low levels of homologous recombination. Appl. Environ. Microbiol. 73:6601-6611.

Didelot, X., and D. Falush. 2007. Inference of bacterial microevolution using multilocus sequence data. Genetics 175:1251-1266.

Didelot, X., and M. C. Maiden. 2010. Impact of recombination on bacterial evolution. Trends Microbiol. 18:315-322.

Falush, D., M. Stephens, and J. K. Pritchard. 2007. Inference of population structure using multilocus genotype data: Dominant markers and null alleles. Mol. Ecol. Notes 7:574-578.

Feil, E. J., B. C. Li, D. M. Aanensen, W. P. Hanage, and B. G. Spratt. 2004. eBURST: Inferring patterns of evolutionary descent among clusters of related bacterial genotypes from multilocus sequence typing data. J. Bacteriol. 186:1518-1530.

Giraffa, G. 2014. Lactobacillus helveticus: Importance in food and health. Front. Microbiol. 5:338.

Giraffa, G., M. Gatti, L. Rossetti, L. Senini, and E. Neviani. 2000. Molecular diversity within Lactobacillus helveticus as revealed by genotypic characterization. Appl. Environ. Microbiol. 66:12591265.

Haubold, B., and R. R. Hudson. 2000. LIAN 3.0: Detecting linkage disequilibrium in multilocus data. Bioinformatics 16:847-848.

Huson, D. H., and D. Bryant. 2006. Application of phylogenetic networks in evolutionary studies. Mol. Biol. Evol. 23:254-267.

Jenkins, J. K., W. J. Harper, and P. D. Courtney. 2002. Genetic diversity in Swiss cheese starter cultures assessed by pulsed field gel electrophoresis and arbitrarily primed PCR. Lett. Appl. Microbiol. 35:423-427.

Jensen, M. P., Y. Ardo, and F. K. Vogensen. 2009. Isolation of cultivable thermophilic lactic acid bacteria from cheeses made with mesophilic starter and molecular comparison with dairy-related $L a c$ tobacillus helveticus strains. Lett. Appl. Microbiol. 49:396-402.

Jolley, K. A., E. J. Feil, M. S. Chan, and M. C. Maiden. 2001. Sequence type analysis and recombinational tests (START). Bioinformatics 17:1230-1231.

Kaleta, P., J. O'Callaghan, G. F. Fitzgerald, T. P. Beresford, and R. P. Ross. 2010. Crucial role for insertion sequence elements in Lactobacillus helveticus evolution as revealed by interstrain genomic comparison. Appl. Environ. Microbiol. 76:212-220. 
Kilpi, E.-R., M. Kahala, J. Steele, A. Pihlanto, and V. Joutsjoki. 2007. Angiotensin I-converting enzyme inhibitory activity in milk fermented by wild-type and peptidase-deletion derivatives of Lactobacillus helveticus CNRZ32. Int. Dairy J. 17:976-984.

Kimura, K., A. Nagasawa, M. Fujii, and Y. Itoh. 2002. Cloning of the pepX gene of Lactobacillus helveticus IF03809 encoding salttolerant X-prolyl dipeptidyl aminopeptidase and characterization of the enzyme. J. Biosci. Bioeng. 93:589-594.

Laukkanen-Ninios, R., X. Didelot, K. A. Jolley, G. Morelli, V. Sangal, P. Kristo, C. Brehony, P. F. Imori, H. Fukushima, A. Siitonen G. Tseneva, E. Voskressenskaya, J. P. Falcao, H. Korkeala, M. C. Maiden, C. Mazzoni, E. Carniel, M. Skurnik, and M. Achtman. 2011. Population structure of the Yersinia pseudotuberculosis complex according to multilocus sequence typing. Environ. Microbiol. 13:3114-3127.

Liu, W., Q. Bao, Jirimutu, M. Qing, Siriguleng, X. Chen, T. Sun, M. Li, J. Zhang, J. Yu, M. Bilige, and H. Zhang. 2012. Isolation and identification of lactic acid bacteria from Tarag in Eastern Inner Mongolia of China by 16S rRNA sequences and DGGE analysis. Microbiol. Res. 167:110-115.

Liu, W., Z. Sun, J. Zhang, W. Gao, W. Wang, L. Wu, T. Sun, W. Chen, X. Liu, and H. Zhang. 2009. Analysis of microbial composition in acid whey for dairy fan making in Yunnan by conventional method and 16S rRNA sequencing. Curr. Microbiol. 59:199-205.

Maiden, M. C., J. A. Bygraves, E. Feil, G. Morelli, J. E. Russell, R. Urwin, Q. Zhang, J. Zhou, K. Zurth, and D. A. Caugant. 1998. Multilocus sequence typing: A portable approach to the identification of clones within populations of pathogenic microorganisms. Proc. Natl. Acad. Sci. USA 95:3140-3145.

Nakamura, Y., N. Yamamoto, K. Sakai, A. Okubo, S. Yamazaki, and T. Takano. 1995. Purification and characterization of angiotensin I-converting enzyme inhibitors from sour milk. J. Dairy Sci. 78:777-783.

Picozzi, C., G. Bonacina, I. Vigentini, and R. Foschino. 2010. Genetic diversity in Italian Lactobacillus sanfranciscensis strains assessed by multilocus sequence typing and pulsed-field gel electrophoresis analyses. Microbiology 156:2035-2045.

Ramachandran, P., D. W. Lacher, E. A. Pfeiler, and C. A. Elkins. 2013. Development of a tiered multilocus sequence typing scheme for members of the Lactobacillus acidophilus complex. Appl. Environ. Microbiol. 79:7220-7228.

Rozas, J., J. C. Sanchez-DelBarrio, X. Messeguer, and R. Rozas. 2003. DnaSP, DNA polymorphism analyses by the coalescent and other methods. Bioinformatics 19:2496-2497.
Salvetti, E., S. Torriani, and G. E. Felis. 2012. The genus Lactobacillus: A taxonomic update. Probiotics Antimicrob Proteins 4:217-226.

Slattery, L., J. O'Callaghan, G. F. Fitzgerald, T. Beresford, and R. P. Ross. 2010. Invited review: Lactobacillus helveticus - A thermophilic dairy starter related to gut bacteria. J. Dairy Sci. 93:44354454.

Su, M. S., P. L. Oh, J. Walter, and M. G. Ganzle. 2012. Intestinal origin of sourdough Lactobacillus reuteri isolates as revealed by phylogenetic, genetic, and physiological analysis. Appl. Environ. Microbiol. 78:6777-6780.

Sun, Z., W. Liu, W. Gao, M. Yang, J. Zhang, L. Wu, J. Wang, B. Menghe, T. Sun, and H. Zhang. 2010a. Identification and characterization of the dominant lactic acid bacteria from kurut: The naturally fermented yak milk in Qinghai, China. J. Gen. Appl. Microbiol. 56:1-10.

Sun, Z., W. Liu, J. Zhang, J. Yu, W. Zhang, C. Cai, B. Menghe, T. Sun, and H. Zhang. 2010b. Identification and characterization of the dominant lactobacilli isolated from koumiss in China. J. Gen. Appl. Microbiol. 56:257-265.

Sun, Z., J. Yu, T. Dan, W. Zhang, and H. Zhang. 2014. Phylogenesis and evolution of lactic acid bacteria. Pages 1-101 in Lactic Acid Bacteria Fundamentals and Practice. H. Zhang and Y. Cai, ed. Springer, New York, NY.

Tanigawa, K., and K. Watanabe. 2011. Multilocus sequence typing reveals a novel subspeciation of Lactobacillus delbrueckii. Microbiology 157:727-738.

Wakai, T., T. Shinoda, N. Uchida, M. Hattori, Y. Nakamura, T. Beresford, R. P. Ross, and N. Yamamoto. 2013. Comparative analysis of proteolytic enzymes need for processing of antihypertensive peptides between Lactobacillus helveticus CM4 and DPC4571. J. Biosci. Bioeng. 115:246-252.

Xu, H., Z. Sun, W. Liu, J. Yu, Y. Song, Q. Lv, J. Zhang, Y. Shao, B. Menghe, and H. Zhang. 2014. Multilocus sequence typing of Lactococcus lactis from naturally fermented milk foods in ethnic minority areas of China. J. Dairy Sci. 97:2633-2645.

Yamamoto, N., M. Maeno, and T. Takano. 1999. Purification and characterization of an antihypertensive peptide from a yogurt-like product fermented by Lactobacillus helveticus CPN4. J. Dairy Sci. 82:1388-1393

Zhao, W., Y. Chen, Z. Sun, J. Wang, Z. Zhou, T. Sun, L. Wang, W. Chen, and H. Zhang. 2011. Complete genome sequence of Lactobacillus helveticus H10. J. Bacteriol. 193:2666-2667. 